\title{
Cervical Spine
}

OPEN

\section{Thermal Stimulation Changes Diffusivity of the Spinothalamic Tract}

\author{
Joanne C. Lin, PhD, Christina Mueller, MS, and Jarred W. Younger, PhD
}

Study Design. An experimental study.

Objective. This study aimed to investigate task-dependent changes in fractional anisotropy (FA) within the spinal cord during painful stimulation.

Summary of Background Data. Earlier experiments by Mandl et al (2008, 2013) used non-invasive functional diffusion tensor imaging (fDTI) to detect white matter fibers that were active during functional tasks. In two studies, it was observed that FA of involved white matter tracts exhibited repeatable taskrelated increases. In this study, we attempted to extend the fDTI work in the spinal cord.

Methods. Twenty-three healthy, right-handed men (mean age 22 yrs, standard deviation $[S D]=4$ ) were invited to participate in this study. Diffusion-weighted images were collected over spinal levels C2 to T4 during a painful thermal stimulus applied to the left thenar eminence. In order to investigate task-related activity, FA values within the contralateral (right) spinothalamic tract were analyzed using a generalized estimating equations (GEE) procedure. As a control, we also examined activity in the ipsilateral and contralateral corticospinal tracts, which are not considered to be involved in nociception.

Results. Significant task-related decreases in FA were observed in the right spinothalamic tract at vertebral levels C2-C5 (Wald $\left.X^{2}(1)=17.754, P<0.001\right)$. There was no change in control regions at levels $\mathrm{C} 7-\mathrm{T} 2$ of the same tract, which are located below the level of input from dermatome C6, Wald

From the Department of Psychology, University of Alabama at Birmingham, University Boulevard, Birmingham, Alabama.

Acknowledgment date: July 13, 2017. First revision date: October 3, 2017. Acceptance date: October 16, 2017.

The manuscript submitted does not contain information about medical device(s)/drug(s).

This work was supported by funds from the Comprehensive Neuroscience Center at UAB.

No relevant financial activities outside the submitted work.

This is an open access article distributed under the terms of the Creative Commons Attribution-Non Commercial-No Derivatives License 4.0 (CCBYNC-ND), where it is permissible to download and share the work provided it is properly cited. The work cannot be changed in any way or used commercially without permission from the journal.

Address correspondence and reprint requests to Joanne C. Lin, PhD, Department of Psychology, University of Alabama at Birmingham, 233 Campbell Hall, 1300 University Boulevard, Birmingham, AL 35294;

E-mail: jclin@uab.edu

DOI: 10.1097/BRS.0000000000002472
$X^{2}(1)=0.185, P=0.667$. Results in all other regions assessed, that is, the left spinothalamic tract and bilateral corticospinal tract, were also not significant $(P>0.05)$.

Conclusion. The current findings suggest that task-related changes in FA associated with the transmission of pain signals along the spinal cord can be detected using fDTI. We observed decreased FA values in the contralateral (right) spinothalamic tract following painful stimulation, while no such activity was apparent in control regions.

Key words: diffusion tensor imaging, fractional anisotropy, magnetic resonance imaging, spine, thermal pain.

Level of Evidence: 5

Spine 2018;43:E697-E702

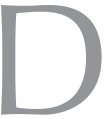

escribing connectivity of the central nervous system (CNS) during tasks is a high priority for human neuroscience researchers. In the magnetic resonance imaging (MRI) environment, several techniques have been developed for defining functional brain networks. These approaches typically examine the associations in blood-oxygen-level-dependent (BOLD) activity between discrete regions of gray matter. While highly informative, BOLDbased connectivity analyses do not provide information about the activity of the axons that underlie CNS connectivity. Without assessing axonal function, it is not possible to determine if two regions of gray matter with correlated activity are directly or indirectly connected. An imaging approach that allows the measurement of axon activity during functional tasks could be an important complement to existing measures of gray matter connectivity.

To address the lack of axonal activity measurements in MRI, Mandl et $a l^{1,2}$ explored a functional diffusion tensor imaging (fDTI) method to identify white matter fibers that are active during functional tasks. In the two studies by Mandl et al, it was observed that fractional anisotropy (FA) of involved white matter tracts exhibited repeatable task-related increases. These changes were observed in optic radiations during a visual task, and the thalamo-cortical tract during tactile stimulation. The fDTI response had unique temporal characteristics, requiring 1 minute to reach peak levels and 3 minutes to return to baseline. The time course of the fDTI signal makes it easily separable from noise and other functional signals, including the BOLD response. . $^{3,4}$ 
In this study, we attempted to extend the fDTI work in two ways. First, we moved the data acquisition to the spinal cord to measure spinal rather than supraspinal task-related activity. Second, we employed a noxious (thermal pain) stimulus to examine nociceptive processing. A scan that could identify abnormal nociceptive processing in the spinal cord would be highly desirable to clinical pain researchers. We hypothesized that the thermal stimulus would cause FA increases in the contralateral spinothalamic tract, as that tract decussates at the level of entry and carries nociceptive information to the brain. ${ }^{5}$

FA describes the directionality of water diffusion, and ranges from 0 to 1 . Values close to 0 indicate unrestricted diffusion in all directions, whereas values close to 1 indicate diffusion along a primary axis and restricted along others. Axons of the human spinal cord are arranged in parallel fiber "bundles." Although axons leave and enter the spinal cord at all levels, the majority of spinal cord activity is relayed through electrical signals traveling either towards (afferent) or away from (efferent) the brain. The axon bundles restrict diffusion in the spinal cord, and this is reflected by FA values closer to 1 . Changes in FA values in response to experimental stimuli may be attributable to physical changes in the environment, such as morphological changes in glial cells to accommodate signal transmission. ${ }^{6}$ Morphological changes in spinal cord neurons and glia that align axons more tightly along a primary axis may facilitate more efficient transmission of afferent electrical signals. Therefore, task-related increases in FA values are plausible. However, the directionality of our hypothesis was based on these theoretical considerations rather than previous findings. Thus, our statistical analyses were two-tailed and were equally powered to detect increases or decreases in FA values. As a control, we also examined activity in the ipsilateral and contralateral corticospinal tracts, which are not considered to be involved in nociception.

\section{MATERIALS AND METHODS}

\section{Participants}

Twenty-three healthy, right-handed men (mean age 22 yrs, standard deviation $[\mathrm{SD}]=4$ ) were invited to participate in the study. Inclusion was limited to male participants as the female menstrual cycle may influence experimental pain sensitivity, and studies have shown differential brain-related activation patterns across the menstrual cycle. ${ }^{7}$ None of the participants had medical conditions or were taking medications. All study procedures were approved by the Institutional Review Board at the University of Alabama at Birmingham (UAB), and written informed consent was obtained from all participants before initiating the study protocol.

\section{Study Procedure}

Thermal pain was elicited with the Medoc Pathway (Medoc Ltd, Ramat Yishai, Israel) MRI-compatible Advanced Thermal Stimulator $30 \times 30 \mathrm{~mm}$ probe. Prior to scanning, participants underwent a thresholding protocol to determine

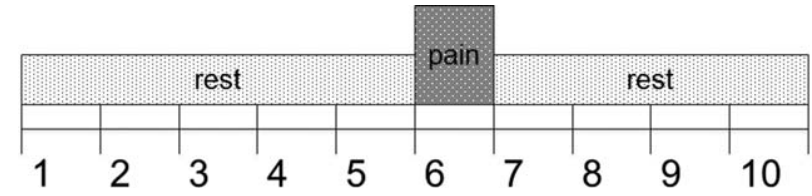

Figure 1. Functional task. Each run was approximately 10 minutes. The run was conducted four times, for a total task time of 40 minutes.

appropriate thermal stimulation levels for the scan. The target pain level was seven out of an 11-point scale, indicating moderately high pain. To determine the temperature required to elicit that level of pain, the probe was attached to the thenar eminence of the right hand. The thermode temperature was increased from a baseline of $32.0^{\circ} \mathrm{C}$ at a rate of $5.0^{\circ} \mathrm{C} / \mathrm{s}$ to the destination temperature, held for 54 seconds, and then decreased at a rate of $5.0^{\circ} \mathrm{C} / \mathrm{s}$ back to baseline. Testing began at an average temperature of $45.5^{\circ} \mathrm{C}$ and, depending on the subjective pain rating, temperatures increased or decreased in increments of $0.5^{\circ} \mathrm{C}$. After each stimulus, participants were asked to verbally rate their pain on an 11-point numerical rating scale (0 being "no pain" and 10 being "worst pain imaginable"). Testing was repeated until the participant reported two ratings of 7 for a given temperature in two separate cycles. For safety, temperatures did not exceed $48.0^{\circ} \mathrm{C}$.

Once the stimulus temperature was determined, participants were placed in the scanner. For the study task, the thermode was placed on the thenar eminence of the left hand. Participants completed four nociceptive stimulus presentations at their predetermined 7/10 temperature. Each run consisted of 5 minutes of baseline, 1 minute of thermal stimulation, and 4 minutes of recovery (Figure 1). After the task was completed, participants were asked to verbally rate their average pain over the four stimulus presentations using the 11-point scale.

\section{Image Acquisition}

Data were collected at the Civitan International Neuroimaging Laboratory at UAB, using a 3.0T Siemens Magnetom Prisma System (Siemens Medical Solutions, Erlangen, Germany) and a 64-channel head/neck with 32-channel spine coil. Head coil elements 1 to 4 were not used during imaging to minimize motion artifacts from the internal carotid arteries.

A T2-weighted turbo spin-echo (TSE) scan covering the first cervical vertebra to the fourth thoracic vertebra was acquired for anatomical reference using the following parameters: sagittal slices; repetition time $(\mathrm{TR})=10580 \mathrm{~ms}$; $\mathrm{ms}$; echo time $(\mathrm{TE})=111 \mathrm{~ms}$; field of view $(\mathrm{FOV})=$ $220 \times 220 \mathrm{~mm}$; matrix $=269 \times 384$; voxel resolution $=$ $0.6 \times 0.6 \times 0.8 \mathrm{~mm}$.

For the functional task, diffusion-weighted images were collected over the same spinal levels (C2-T4) using a readout-segmented, multi-shot echo planar (RESOLVE) sequence $^{8}$ and the following parameters: sagittal slices; $\mathrm{TR}=1600 \mathrm{~ms} ; \quad \mathrm{TE}=52 \mathrm{~ms} ; \quad \mathrm{FOV}=506 \times 1000 \mathrm{~mm} ;$ 
matrix $=82 \times 162 ; 12$ slices; $10 \%$ gap; GRAPPA factor $=2$; voxel resolution $=1.5 \times 1.5 \times 1.5 \mathrm{~mm}$. Diffusion gradients were applied along six non-collinear directions using $b$-values of 0 and $700 \mathrm{~s} / \mathrm{mm}^{2}$.

During the functional task, participants received a thermal stimulus to the left thenar eminence (dermatome C6) for 54 seconds between rest intervals (baseline temperature of $32^{\circ} \mathrm{C}$ ). A total of 40 acquisitions were made for the 40-minute task, plus four dummy acquisitions at the beginning of the scan to eliminate possible scanner onset effects (e.g., gradient heating).

\section{Image Processing}

Data were analyzed using Spinal Cord Toolbox version 2.2 (https://sourceforge.net/projects/spinalcordtoolbox). ${ }^{9}$ First, the T2-weighted scan was segmented using a fully automated method, PropSeg ${ }^{10}$ to produce a binary mask of the spinal cord. Next, the C2 and T3 vertebrae were manually identified, and a vertebral landmark mask was generated. The structural image was then straightened using the binary mask, and nonlinearly registered to the T2-weighted MNIPoly-AMU template (resolution $=0.5 \times 0.5 \times 0.5 \mathrm{~mm}$ ) based on vertebral landmarks. ${ }^{11}$

Diffusion data were motion corrected using an iterative 2D retrospective motion correction and intensity-based outlier detection to minimize the influence of physiological noise. ${ }^{12}$ The data were registered to the structural image using a nonlinear deformation constrained to the axial plane. The diffusion-to-structural and structural-to-template deformation fields were then concatenated to enable forward transformation of the diffusion-weighted images to template space and the inverse transformation of white matter tracts in template space back to individual space. For quality control, all images were visually inspected at each transformation step.

Diffusion tensors were computed for each run using DTIFIT from FMRIB's Diffusion Toolbox. ${ }^{13}$ Tracts of interest were identified from the white matter atlas ${ }^{14}$ and FA values were extracted using a maximum a posteriori method, which overcomes biases associated with manual delineation and partial volume effects. ${ }^{15}$ FA was extracted from the bilateral spinothalamic tracts (STT) and bilateral corticospinal tracts (CST) in spinal levels above and below the stimulus dermatome. The "above" region of interest included the $\mathrm{C} 2-\mathrm{C} 5$ vertebrae (corresponding to C3-C5 and partial C6 spinal levels), and the "below" region of interest included the $\mathrm{C} 7-\mathrm{T} 2$ vertebrae (corresponding to partial C8 through to T2 spinal levels).

The fDTI technique assumes that task-related changes should be represented along the entire tract so data are aggregated along all voxels, which provides a gain in signalto-noise ratio, as well as statistical power. It also reduces the potential confounding impact of task-related activity changes within neighboring gray matter voxels. Therefore, this approach has the potential to complement conventional network analyses by allowing us to visualize activity that connects gray matter regions.

\section{Statistical Analyses}

Statistical analyses were conducted in IBM SPSS Statistics 22 (IBM Corp). FA values from each tract segment of interest were person-centered. The centered values were used as outcomes in statistical analyses.

The main predictor of interest was task condition (baseline $v$ s. stimulus). Baseline was defined as acquisitions 1 to 5 of each run, and stimulus was defined as acquisitions 6 to 8 . The 3-minute stimulus period was defined a priori based on previous reports of a 3-minute fDTI response to tasks. ${ }^{1,2}$ The final two acquisitions from each run were used as a recovery period to allow FA values to return to baseline levels, and were not included in analyses. Because each run was repeated four times, each participant contributed a total 20 FA measurements during the baseline condition and 12 measurements during the pain condition.

\section{Generalized Estimating Equations}

In order to determine whether FA values in tracts of interest differed between the baseline and pain condition of the task sequence, we used a generalized estimating equations (GEE) procedure. This procedure is appropriate in studies involving a high number of longitudinal assessments where repeated measures are "nested" within each participant. GEEs are designed to estimate an average population response (rather than individual responses) and require fewer assumptions and prior information than do linear mixed models. We used a GEE with the following parameters: subject nesting index $=$ subject ID, repeated measures index $=$ time, within-subject working correlation matrix $=$ autoregressive $(\mathrm{AR} 1)$, model type $=$ linear, probability distribution $=$ normal, link function $=$ identity, parameter estimation method $=$ maximum likelihood. Task condition (baseline $v s$. time) was the main within-subjects predictor of interest in the model. Time was included in the model as a covariate to account for scanner signal drift. The conditionby-time interaction was also included in the model. To account for multiple comparisons, a false discovery rate of 0.05 was used to adjust thresholds for determining statistical significance over eight analyses (equivalent to uncorrected $P<0.0184)$.

Because this fDTI scan approach had not been previously performed on the spinal cord, we did not have preliminary information on effect size and variance to conduct a sample size calculation. Therefore, this study was conducted to establish parameters for power calculations in future studies. We set a target recruitment goal of at least 20 study completers in order to be consistent with other functional neuroimaging tasks using other sequences.

\section{RESULTS}

Data from four participants were excluded from analyses due to visible movement during acquisition $(\mathrm{n}=3)$ and anatomical abnormality $(\mathrm{n}=1)$, leaving data from 19 participants. For two of those individuals, data were not obtained below the second thoracic vertebra. For one participant, the final run was excluded due to excessive movement. 


\begin{tabular}{|c|c|c|c|c|c|c|}
\hline & & \multicolumn{5}{|c|}{ FA } \\
\hline & & Baseline & Pain & Wald $X^{2}$ & $d f$ & $P$ \\
\hline \multirow[t]{2}{*}{ Right STT } & $\mathrm{C} 2-\mathrm{C} 5$ & $0.800(0.079)$ & $0.790(0.079)$ & 17.754 & 1 & $<0.001$ \\
\hline & $\mathrm{C} 7-\mathrm{T}^{*}$ & $0.800(0.069)$ & $0.791(0.068)$ & 0.185 & 1 & 0.667 \\
\hline \multirow[t]{2}{*}{ Left STT } & $\mathrm{C} 2-\mathrm{C} 5$ & $0.794(0.071)$ & $0.796(0.077)$ & 0.013 & 1 & 0.909 \\
\hline & $\mathrm{C} 7-\mathrm{T}^{*}$ & $0.801(0.074)$ & $0.803(0.075)$ & 0.847 & 1 & 0.357 \\
\hline \multirow[t]{2}{*}{ Right CST } & $\mathrm{C} 2-\mathrm{C} 5$ & $0.768(0.074)$ & $0.764(0.080)$ & 1.689 & 1 & 0.194 \\
\hline & $\mathrm{C} 7-\mathrm{T}^{*}$ & $0.771(0.078)$ & $0.770(0.076)$ & 0.021 & 1 & 0.884 \\
\hline \multirow[t]{2}{*}{ Left CST } & $\mathrm{C} 2-\mathrm{C} 5$ & $0.780(0.068)$ & $0.776(0.070)$ & 0.561 & 1 & 0.454 \\
\hline & C7-T2* & $0.779(0.067)$ & $0.774(0.067)$ & 0.194 & 1 & 0.660 \\
\hline
\end{tabular}

The average temperature for nociceptive stimuli was $45.8 \pm 0.6^{\circ} \mathrm{C}$ (range $=45-47^{\circ} \mathrm{C}$ ), which elicited an average prescan pain rating of $7.2 \pm 1.1$. The average postscan rating was $6.7 \pm 0.9$.

\section{Task-Related Changes in FA}

Results from GEE analyses on centered FA values are displayed in Table 1. Condition-by-time interactions and main effects of time were not significant in any of the assessed regions $(P>0.05)$. Therefore, we report results for main effects of task condition. Significant task-related changes in FA were observed in the right spinothalamic tract at vertebral levels C2-C5 (Wald $X^{2}(1)=17.754, P<0.001$ ), with values decreasing from 0.800 during baseline to 0.790 following the nociceptive stimulus. There was no change in control regions at levels C7-T2 of the same tract, which are located below the level of input from dermatome C6, Wald $X^{2}(1)=0.185, P=0.667$ (Figure 2). Main effects in all other regions assessed, i.e., the left spinothalamic tract and bilateral corticospinal tract, were also not significant $(P>0.05)$.

\section{DISCUSSION}

The primary aim of this study was to investigate taskdependent changes in FA within the spinal cord during nociceptive stimulation. A thermal stimulus was applied to the left thenar eminence of the hand, corresponding to dermatome C6. We observed task-related changes in FA in the contralateral spinothalamic tract, at and above the $\mathrm{C} 6$ vertebral level. Significant task-related activity was not observed in the tract below the C6 level, and was not observed in the ipsilateral spinothalamic tract or bilateral corticospinal tracts.

Based on the original method and outcomes reported by Mandl et $a l,{ }^{1,2}$ we hypothesized that the fDTI response function would require approximately 4 minutes to resolve, and would be represented by increased FA. Consistent with Mandl's work, the response peaked at 1 minute after stimulus onset, and returned to baseline after an additional
2 minutes (see Figure 3). However, in contrast to previous work, we observed FA to decrease during thermal stimulation. Two major methodological differences may explain the divergent results: stimulus modality and location. While Mandl et al used innocuous tactile and visual tasks, we delivered a nociceptive stimulus. Nociceptive stimulation may yield a different response than other sensory modalities, particularly in the spine where ascending nociceptive information can be suppressed by descending pain modulatory mechanisms. ${ }^{16}$ Future fDTI work will need to examine spinal cord activity to non-noxious tasks, as well as supraspinal processing of pain, to determine under what conditions FA exhibits task-related increases versus decreases.

A related point that will require clarification in future studies is the magnitude of a response that is physiologically meaningful. The task-related decrease from 0.800 to 0.790 represents a percent change of only $2.5 \%$. Although this is small, the difference was statistically significant and is attributable to the experimental stimulus. We note that large, task-related changes in FA would not be expected, as the majority of the FA signal is driven by the structure of the spinal cord tracts that is invariable over a short timeframe.

We do not know what physiological processes underlie task-related changes in FA. Mandl et al $^{1,2}$ suggested that FA task reactivity was due to transient morphological changes of glial cells. This hypothesis is supported by recent work by Micu et al, ${ }^{6}$ who posit the existence of an axo-myelinic synapse that allows neurons to communicate with their myelinating glial cells. This synapse allows the solicitation of metabolic support, and signals oligodendrocytes to adjust the properties of its myelin in a dynamic, activity-dependent manner. The authors showed that by electrically stimulating optic nerve axons ex vivo, they could cause a thickening of the myelin sheath as revealed by two-photon microscopy. The thickening was due to increased spacing between myelin lamellae. While myelin change is an intriguing possible explanation for the fDTI signal, additional work will be needed to confirm its role. 
Figure 2. Ten-point time courses in the right spinothalamic tract at vertebral levels C2-C5 (top; corresponding to spinal levels $\mathrm{C} 3-\mathrm{C} 5$ and partial C6) and vertebral levels (bottom; corresponding to spinal levels partial C8) through T2 (bottom). Time courses were created as in Figure 2. Error bars represent 95\% confidence intervals.

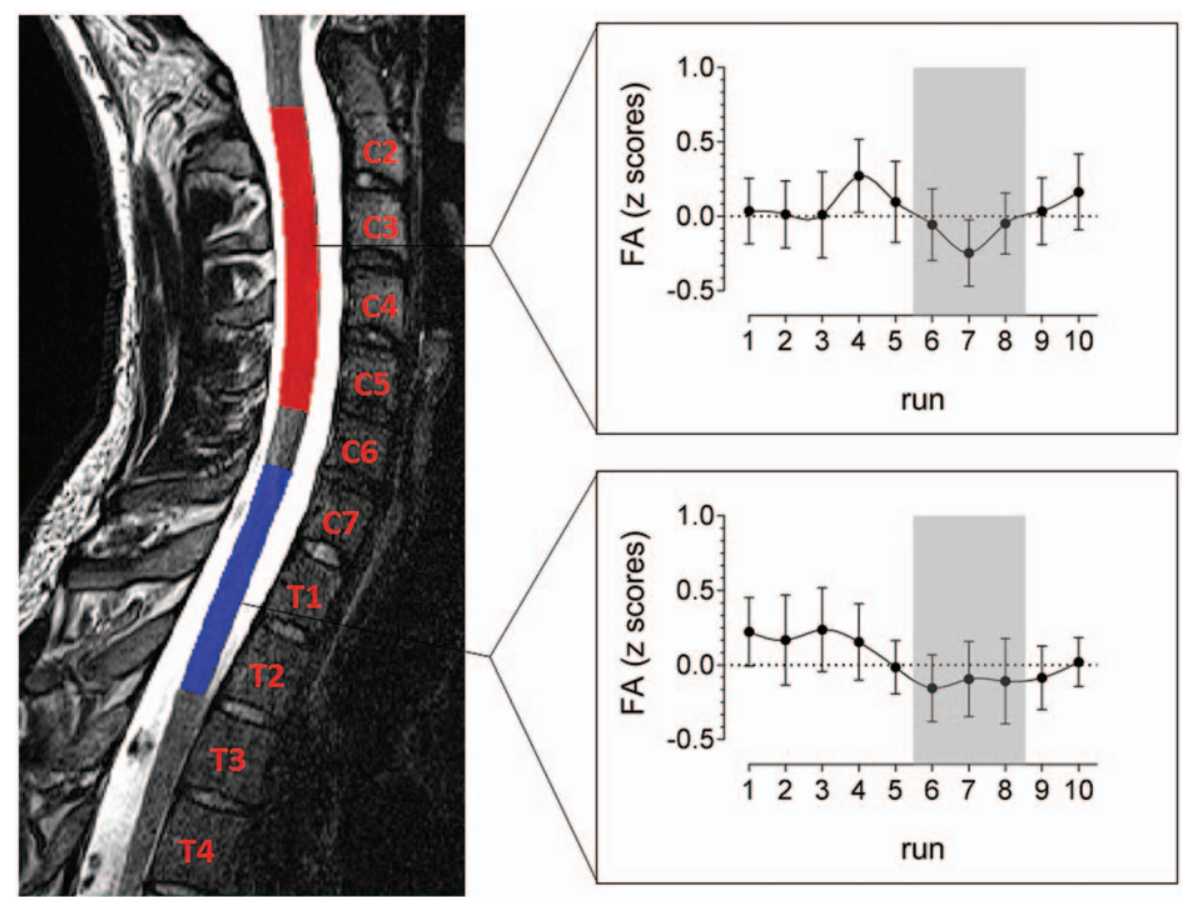

\section{Limitations}

There are limitations that reduce the interpretability of our findings, and the general use of fDTI as a neuroimaging tool. First, the time required to observe a complete response (3-4 min) is lengthier than BOLD and other functional acquisitions. The time required for a single response limits the number of stimulus presentations, as well as the number of tasks that can be comfortably performed. As a result, signal-to-noise is not optimal, and control tasks may have to be omitted from protocols.

Second, in order to reduce the time required to obtain each fDTI volume, we limited the acquisition to seven volumes (b0 and six non-collinear directions). While this number of directions is the minimum needed for tensor fitting and FA calculation, it is not optimal in the brain where complex, crossing fibers need to be accurately

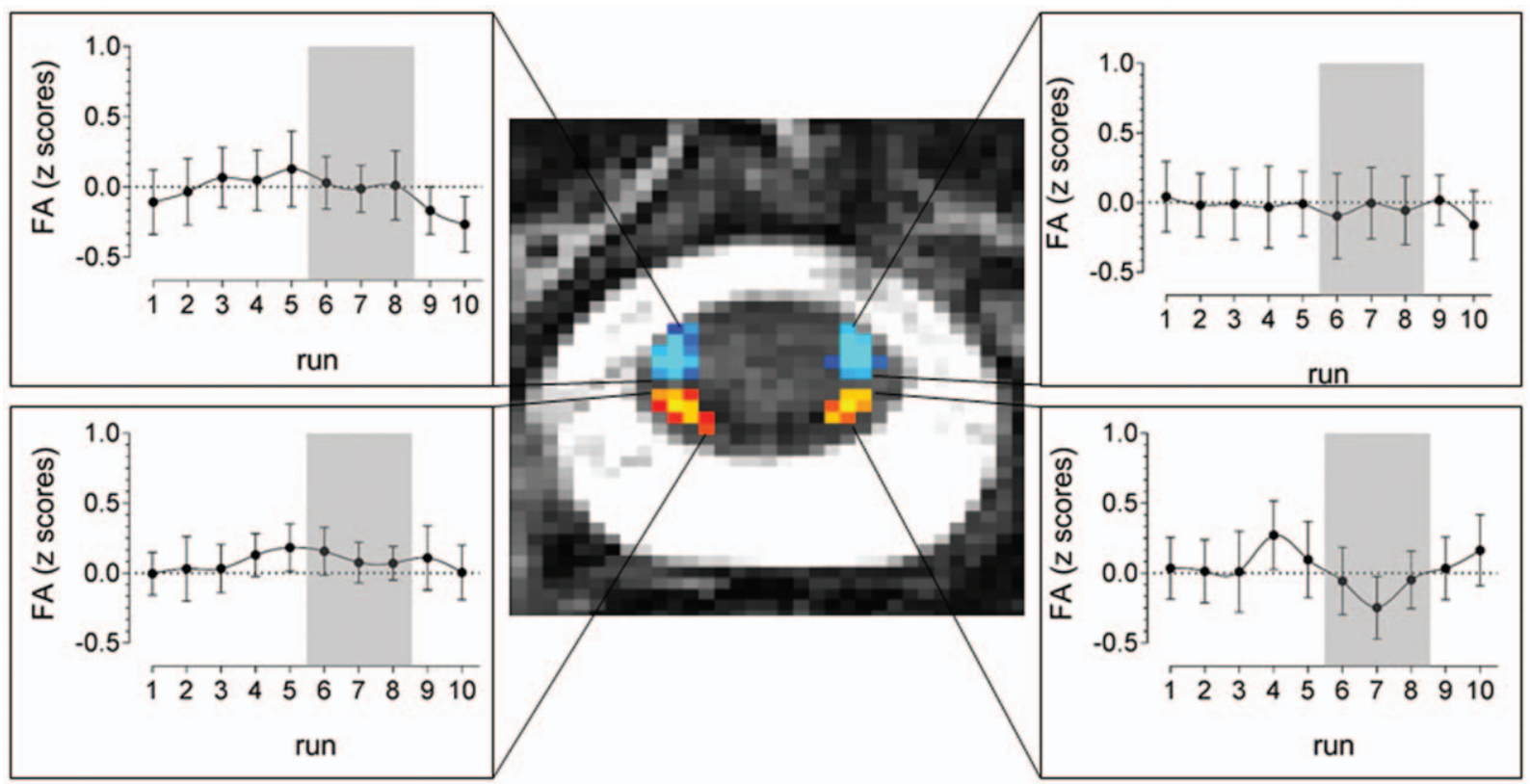

Figure 3. Ten-point time courses in left and right spinothalamic (red) and corticospinal (blue) tracts from vertebral levels C2-C5 (spinal levels C3-C5 and partial C6). Individual raw scores from 40 time points were smoothed with a 3-point moving average and converted into z-scores using each participant's mean and standard deviation. Ten-point time courses were created for each individual by calculating the median of four measurements for each run. The time courses were then averaged across participants and a cubic spline curve was fitted to the data. Error bars represent 95\% confidence intervals. 
estimated. This issue is mitigated in the spinal cord where the fibers mainly track in only one direction. Even with an acquisition of only seven volumes per time point, 1 minute is required per time point, which yields fairly poor temporal resolution.

Third, diffusion-weighted acquisitions are sensitive to motion artifacts caused by cardiac pulsation and respiratory cycles, which may complicate signal detection. Future studies may use cardiac gating during acquisition, or collect physiological noise signals to enable retrospective correction.

The sample used in this pilot study was intended to minimize heterogeneity. Therefore, it is not known how the results would generalize to older and younger individuals, women, and individuals with various pain, neuropathic, and other medical conditions. The observed response is not necessarily typical of the general population, and future studies may determine sex and other biological differences. In addition to increasing generalizability, future studies using this sequence should test various noxious and nonnoxious tasks to further understand the implications of the observed FA changes. Conditions involving abnormal pain processing, spinal damage, or sustained nociceptive inputs may also be investigated.

\section{CONCLUSION}

The current findings suggest that task-related changes in FA associated with the transmission of nociceptive signals along the spinal cord can be detected using fDTI. We observed decreased FA values in the contralateral (right) spinothalamic tract following nociceptive stimulation, while no such activity was apparent in control regions. Future studies may incorporate larger sample sizes, improved spatial and temporal resolution, optimizing baseline and task timing. Additional controls tasks could be incorporated to validate and extend these findings; for example, an active motor (e.g., finger tapping) to investigate response in the corticospinal tracts and/or a vibrational tactile task on the upper limb to explore the fasciculus cuneatus. Future studies will also need to delineate the physiological mechanisms underlying changes in water diffusivity along white matter tracts in various central nervous system regions, as some of these may differ. The fDTI acquisition is relatively simple and could be employed at any MR scanner capable of conducting DTI scans. Using the fDTI technique to non-invasively visualize and quantify activity in white matter tracts in various settings could further our understanding of CNS connectivity.

\section{Key Points}

Describing connectivity of the CNS during tasks is a high priority for human neuroscience researchers; however, current MRI methods do not provide information about the activity of axons that underlie CNS connectivity.

$\square$ fDTI was used to investigate changes in FA within white matter tracts of the spinal cord during nociceptive processing.

- Significant task-related decreases in FA were observed in the right spinothalamic tract. These results suggest that task-related changes in FA associated with the transmission of pain signals along the spinal cord can be detected using fDTI.

\section{References}

1. Mandl RC, Schnack HG, Zwiers MP, et al. Functional diffusion tensor imaging at 3 Tesla. Front Hum Neurosci 2013;7: 817.

2. Mandl RC, Schnack HG, Zwiers MP, et al. Functional diffusion tensor imaging: measuring task-related fractional anisotropy changes in the human brain along white matter tracts. PLoS One 2008;3:e3631.

3. Yarkoni T, Barch DM, Gray JR, et al. BOLD correlates of trial-bytrial reaction time variability in gray and white matter: a multistudy fMRI analysis. PLoS One 2009;4:e4257.

4. Fraser LM, Stevens MT, Beyea SD, et al. White versus gray matter: fMRI hemodynamic responses show similar characteristics, but differ in peak amplitude. BMC Neurosci 2012;13: 91.

5. Purves D, Augustine GJ, Fitzpatrick D, et al. Central pain pathways: the spinothalamic tract.Neuroscience, 3rd ed. Sunderland, MA: Sinauer Associates; 2004.

6. Micu I, Plemel JR, Lachance C, et al. The molecular physiology of the axo-myelinic synapse. Exp Neurol 2016;276:41-50.

7. Iacovides S, Avidon I, Baker FC. Does pain vary across the menstrual cycle? A review. Eur J Pain 2015;19:1389-405.

8. Porter DA, Heidemann RM. High resolution diffusion-weighted imaging using readout-segmented echo-planar imaging, parallel imaging and a two-dimensional navigator-based reacquisition. Magn Reson Med 2009;62:468-75.

9. Cohen-Adad J, De Leener B, Benhamou M, et al., editors. Spinal Cord Toolbox: An Open-Source Framework for Processing Spinal Cord MRI data. Proceedings of the 20th Annual Meeting of OHBM, Hamburg, Germany; 2014.

10. De Leener B, Kadoury S, Cohen-Adad J. Robust, accurate and fast automatic segmentation of the spinal cord. NeuroImage 2014;98: 528-36.

11. Fonov VS, Le Troter A, Taso M, et al. Framework for integrated MRI average of the spinal cord white and gray matter: the MNIPoly-AMU template. NeuroImage 2014;102:817-27.

12. Xu J, Shimony JS, Klawiter EC, et al. Improved in vivo diffusion tensor imaging of human cervical spinal cord. Neurolmage 2013;67:64-76.

13. Smith SM, Jenkinson M, Woolrich MW, et al. Advances in functional and structural MR image analysis and implementation as FSL. NeuroImage 2004;23 (Suppl):S208-19.

14. Benhamou M, Fonov V, Taso M, et al., editors. Atlas of whitematter tracts in the human spinal cord. ISMRM-ESMRMB Annual Meeting 2014; 2014.

15. Levy S, Benhamou M, Naaman C, et al. White matter atlas of the human spinal cord with estimation of partial volume effect. NeuroImage 2015;119:262-71.

16. Vanegas H, Schaible H-G. Descending control of persistent pain: inhibitory or facilitatory? Brain Res Brain Res Rev 2004;46: 295-309. 